\title{
Accuracy of ICD-10 Diagnostic Codes to Identify COVID-19 Among Hospitalized Patients
}

J Gen Intern Med 36(8):2532-5 DOI: $10.1007 / \mathrm{s} 11606-021-06936-\mathrm{w}$

(C) Society of General Internal Medicine 2021

\section{BACKGROUND}

Health organizations require rapid, reliable access to data on patients infected with severe acute respiratory syndrome coronavirus 2 (SARS-CoV-2). On April 1, 2020, the Centers for Disease Control and Prevention (CDC) introduced a new International Classification of Disease, $10^{\text {th }}$ revision (ICD10) code U07.1 (COVID-19, virus identified), ${ }^{1}$ together with specific coding guidance regarding its appropriate use. ${ }^{2}$ Government agencies, including the Centers for Medicare and Medicaid Services, use ICD-10 data for ascertainment of COVID-19 hospitalizations, ${ }^{3}$ though little is known about the reliability of the U07.1 code in identifying disease. We sought to determine the performance characteristics of the ICD-10 code U07.1 for identification of COVID-19 illness in a large multicenter health system.

\section{METHODS}

We identified all inpatient encounters during which $\geq 1$ SARSCoV-2 reverse transcription polymerase chain reaction (RTPCR) was performed from April 1 to July 31, 2020, across the Mass General Brigham health system. Patients with $\geq 1$ positive SARS-CoV-2 RT-PCR were denoted to be COVID-19 positive. The agreement between COVID-19 positivity and primary or secondary ICD-10 coding of U07.1 was determined. Performance characteristics (sensitivity, specificity, positive predictive value [PPV], negative predictive value [NPV]) for the ICD-10 code U07.1 were reported overall and across major subgroups. Among COVID-19-positive patients, we performed multivariable logistic regression to identify independent predictors of corresponding ICD-10 coding for COVID-19(sensitivity). The Mass General Brigham Institutional Review Board approved the study protocol. Data management and analysis were performed using STATA (College Station, TX).

Received April 5, 2021

Accepted May 14, 2021

Published online June 7, 2021

\section{RESULTS}

There were 22,633 patient encounters with a discharge date between April 1, 2020, and July 31, 2020, in which $\geq 1$ SARSCoV-2 RT-PCR was obtained during admission. Overall, $66.7 \%, 25.7 \%$, and $7.7 \%$ of encounters had 1,2 , and $>2$ SARS-CoV-2 RT-PCR test(s) performed, respectively (range 1 to 16). Among these encounters, 2210 (9.8\%) were determined to be COVID-19 positive. COVID-19 test-positive patients were older ( $64 \pm 18$ vs. $60 \pm 19$ years) and more likely to be men ( $51.8 \%$ vs. $44.3 \%)$, Hispanic ( $22.3 \%$ vs. $8.3 \%)$, and Black $(21.6 \%$ vs. $10.4 \%)$ as compared with those with all negative RT-PCR results ( $\mathrm{P}<0.001$ for all). ICD-10 diagnostic code U07.1 was coded in 1208 (5.3\%) patients. U07.1 had an overall sensitivity of $49.2 \%$ ( $95 \%$ confidence interval [CI]: $47.1-51.3 \%$ ), specificity of $99.4 \%$ (95\% CI: $99.3-99.5 \%$ ), PPV of $90.0 \%$ (95\% CI: $88.2-91.6 \%$ ), and NPV of $94.8 \%$ (95\% CI: 94.5-95.0\%). Specificity remained high over time: 98.4\% in April, $99.0 \%$ in May, $99.7 \%$ in June, and $99.9 \%$ in July 2020, while sensitivity varied and was lowest in July 2020 (27.9\%). Sensitivity was lower among those age $0-17$ years (14.3\%, 95\% CI: $1.2-70.1 \%)$, although this subset was limited by few encounters $(n=175)$. Similar performance of U07.1 coding was observed across all other major subgroups (Table 1). Earlier months in the pandemic were the only significant independent predictors of higher sensitivity of the ICD-10 diagnostic code U07.1 among COVID-19 testpositive patients (Fig. 1).

\section{DISCUSSION}

Uniform administrative coding for research, disease tracking, and quality improvement is appealing given its widespread use and ease of interoperability across health systems. Reliance on these administrative data will likely remain important for prior COVID-19 disease identification, particularly given expanding interest in identifying legacy effects and postacute sequelae of SARS-CoV-2 (PASC) infection. However, ICD-10 codes in other clinical settings ${ }^{4}$ and for COVID-19related symptoms ${ }^{5}$ are known to be subject to misclassification.

We found sensitivity for U07.1 coding among hospitalized patients undergoing SARS-CoV-2 RT-PCR testing was modest, while specificity was high and approached $100 \%$ over time. Lack of initial awareness or familiarity with ICD-10 coding for COVID-19, in addition to distinctions between test 
Table 1 Performance Characteristics of ICD-10 Code for COVID-19 as Compared with SARS-CoV-2 RT-PCR Positivity

\begin{tabular}{|c|c|c|c|c|c|c|}
\hline \multicolumn{7}{|c|}{ U07.1 ICD-10 code performance characteristics } \\
\hline & \multirow{3}{*}{$\begin{array}{l}\text { \# of patient } \\
\text { encounters } \\
22,633\end{array}$} & \multirow{3}{*}{$\begin{array}{l}\text { \# of encounters with } \geq 1 \\
\text { positive SARS-CoV-2 RT- } \\
\text { PCR, n (\%) } \\
2210(9.8)\end{array}$} & \multicolumn{4}{|c|}{ Point estimate, $\%$ (95\% confidence interval $[\mathrm{CI}]$ ) } \\
\hline & & & Sensitivity & Specificity & PPV & NPV \\
\hline Overall & & & $\begin{array}{l}49.2(47.1- \\
51.3)\end{array}$ & $\begin{array}{l}99.4(99.3- \\
99.5)\end{array}$ & $\begin{array}{l}90.0(88.2- \\
91.6)\end{array}$ & $\begin{array}{l}94.8(94.5- \\
95.0)\end{array}$ \\
\hline \multicolumn{7}{|l|}{ Month } \\
\hline April 2020 & 3827 & $1045(27.3)$ & $\begin{array}{l}45.3(42.3- \\
48.8)\end{array}$ & $\begin{array}{l}98.4(97.9- \\
98.8)\end{array}$ & $\begin{array}{l}91.5(88.7- \\
93.6)\end{array}$ & $\begin{array}{l}82.7(81.4- \\
84.0)\end{array}$ \\
\hline May 2020 & 5982 & $726(12.1)$ & $\begin{array}{l}60.9(57.3- \\
64.4)\end{array}$ & $\begin{array}{l}99.0(98.7- \\
99.2)\end{array}$ & $\begin{array}{l}89.3(86.2- \\
91.7)\end{array}$ & $\begin{array}{l}94.8(94.2- \\
95.4)\end{array}$ \\
\hline June 2020 & 6108 & $292(4.8)$ & $\begin{array}{l}44.9(39.2- \\
50.6)\end{array}$ & $\begin{array}{l}99.7(99.5- \\
99.8)\end{array}$ & $\begin{array}{l}88.5(82.2- \\
92.8)\end{array}$ & $\begin{array}{l}97.3(96.9- \\
97.7)\end{array}$ \\
\hline July 2020 & 6716 & $147(2.2)$ & $\begin{array}{l}27.9(21.2- \\
35.7)\end{array}$ & $\begin{array}{l}99.9(99.8- \\
99.9)\end{array}$ & $\begin{array}{l}85.4(72.0- \\
93.0)\end{array}$ & $\begin{array}{l}98.4(98.1- \\
98.7)\end{array}$ \\
\hline \multicolumn{7}{|l|}{ Age (years) } \\
\hline $0-17$ & 175 & $7(4.0)$ & $\begin{array}{l}14.3(1.2- \\
70.1)\end{array}$ & $100.0(-)$ & $100.0(-)$ & $\begin{array}{l}96.6(92.5- \\
98.5)\end{array}$ \\
\hline $18-34$ & 3116 & $171(5.5)$ & $\begin{array}{l}42.7(35.4- \\
50.3)\end{array}$ & $\begin{array}{l}99.7(99.5- \\
99.9)\end{array}$ & $\begin{array}{l}90.1(81.3- \\
95.0)\end{array}$ & $\begin{array}{l}96.8(96.1- \\
97.3)\end{array}$ \\
\hline $35-64$ & 8917 & $877(9.8)$ & $\begin{array}{l}49.0(45.7- \\
52.3)\end{array}$ & $\begin{array}{l}99.2(99.0- \\
99.4)\end{array}$ & $\begin{array}{l}87.6(84.3- \\
90.2)\end{array}$ & $\begin{array}{l}94.7(94.2- \\
95.2)\end{array}$ \\
\hline$\geq 65$ & 10,425 & $1155(11.1)$ & $\begin{array}{l}50.5(47.6- \\
53.4)\end{array}$ & $\begin{array}{l}99.4(99.3- \\
99.6)\end{array}$ & $\begin{array}{l}91.8(89.4 \\
93.7)\end{array}$ & $\begin{array}{l}94.2(93.7- \\
94.6)\end{array}$ \\
\hline \multicolumn{7}{|l|}{ Gender } \\
\hline Men & 10,191 & $1144(11.2)$ & $\begin{array}{l}48.9(46.0- \\
51.8)\end{array}$ & $\begin{array}{l}99.2(99.0- \\
99.3)\end{array}$ & $\begin{array}{l}88.2(85.4 \\
90.5)\end{array}$ & $\begin{array}{l}93.9(93.4- \\
94.3)\end{array}$ \\
\hline Women & 12,422 & $1066(8.5)$ & $\begin{array}{l}49.5(46.5- \\
52.5)\end{array}$ & $\begin{array}{l}99.6(99.5- \\
99.7)\end{array}$ & $\begin{array}{l}92.0(89.5- \\
93.9)\end{array}$ & $\begin{array}{l}95.5(95.1- \\
95.8)\end{array}$ \\
\hline \multicolumn{7}{|l|}{ Race } \\
\hline White & 16,858 & $1170(6.9)$ & $\begin{array}{l}48.1(45.3- \\
51.0)\end{array}$ & $\begin{array}{l}99.5(99.4- \\
99.6)\end{array}$ & $\begin{array}{l}88.5(85.8- \\
90.8)\end{array}$ & $\begin{array}{l}96.3(96.0- \\
96.5)\end{array}$ \\
\hline Black & 2600 & $478(18.4)$ & $\begin{array}{l}50.0(45.5- \\
54.5)\end{array}$ & $\begin{array}{l}98.8(98.3- \\
99.2)\end{array}$ & $\begin{array}{l}90.5(86.3- \\
93.5)\end{array}$ & $\begin{array}{l}89.8(88.5- \\
90.9)\end{array}$ \\
\hline Other & 3175 & $562(17.7)$ & $\begin{array}{l}50.7(46.6- \\
54.8)\end{array}$ & $\begin{array}{l}99.1(98.7- \\
99.4)\end{array}$ & $\begin{array}{l}92.5(89.0- \\
95.0)\end{array}$ & $\begin{array}{l}90.3(89.2- \\
91.4)\end{array}$ \\
\hline \multicolumn{7}{|l|}{ Ethnicity } \\
\hline Hispanic & 2195 & $492(22.4)$ & $\begin{array}{l}50.8(46.4- \\
55.2)\end{array}$ & $\begin{array}{l}98.5(97.8 .- \\
99.0)\end{array}$ & $\begin{array}{l}90.9(86.9- \\
93.8)\end{array}$ & $\begin{array}{l}87.4(85.8- \\
88.8)\end{array}$ \\
\hline Not Hispanic & 19,410 & $1546(8.0)$ & $\begin{array}{l}48.9(46.4- \\
51.4)\end{array}$ & $\begin{array}{l}99.5(99.4- \\
99.6)\end{array}$ & $\begin{array}{l}88.9(86.6- \\
90.9)\end{array}$ & $\begin{array}{l}95.7(95.4- \\
96.0)\end{array}$ \\
\hline Other/not available & 1028 & $172(16.7)$ & $\begin{array}{l}47.1(39.7- \\
54.6)\end{array}$ & $\begin{array}{l}99.8(99.1- \\
99.9)\end{array}$ & $\begin{array}{l}97.6(90.7- \\
99.4)\end{array}$ & $\begin{array}{l}90.4(88.3- \\
92.1)\end{array}$ \\
\hline \multicolumn{7}{|l|}{ Hospital type } \\
\hline Tertiary care & 14,277 & $1325(9.3)$ & $\begin{array}{l}50.0(47.3- \\
52.7)\end{array}$ & $\begin{array}{l}99.4(99.2- \\
99.5)\end{array}$ & $\begin{array}{l}88.7(86.3- \\
90.8)\end{array}$ & $\begin{array}{l}95.1(94.7- \\
95.5)\end{array}$ \\
\hline Not tertiary care & 8356 & 885 (10.6) & $\begin{array}{l}48.0(44.7- \\
51.3)\end{array}$ & $\begin{array}{l}99.5(99.3- \\
99.6)\end{array}$ & $\begin{array}{l}92.0(89.1- \\
94.1)\end{array}$ & $\begin{array}{l}94.2(93.6- \\
94.7)\end{array}$ \\
\hline \multicolumn{7}{|l|}{ ICU stay } \\
\hline No & 17,514 & $1404(8.0)$ & $\begin{array}{l}47.6(45.0- \\
50.2)\end{array}$ & $\begin{array}{l}99.4(99.3- \\
99.6\end{array}$ & $\begin{array}{l}88.2(85.7- \\
90.4)\end{array}$ & $\begin{array}{l}95.6(95.3- \\
95.9)\end{array}$ \\
\hline Yes & 5119 & $806(15.8)$ & $\begin{array}{l}52.0(48.5- \\
55.4)\end{array}$ & $\begin{array}{l}99.3(99.0- \\
99.5)\end{array}$ & $\begin{array}{l}92.9(90.1- \\
94.9)\end{array}$ & $\begin{array}{l}91.7(90.9- \\
92.5)\end{array}$ \\
\hline \multicolumn{7}{|l|}{$\begin{array}{l}\text { Vital status at } \\
\text { discharge }\end{array}$} \\
\hline Alive & 21,814 & $1917(8.8)$ & $\begin{array}{l}48.5(46.3- \\
50.8)\end{array}$ & $\begin{array}{l}99.4(99.3- \\
99.5)\end{array}$ & $\begin{array}{l}89.0(86.9- \\
90.8)\end{array}$ & $\begin{array}{l}95.2(94.9- \\
95.5)\end{array}$ \\
\hline Deceased & 819 & $293(35.8)$ & $\begin{array}{l}53.6(47.8- \\
59.2)\end{array}$ & $\begin{array}{l}98.9(97.5- \\
99.5)\end{array}$ & $\begin{array}{l}96.3(92.0- \\
98.3)\end{array}$ & $\begin{array}{l}79.3(76.0- \\
82.2)\end{array}$ \\
\hline
\end{tabular}

positivity and clinical disease, may account for lower sensitivity. Lags in coding after hospital discharge and shifts in routine testing practices among hospitalized patients may also partially explain variable sensitivity. The robust specificity of ICD-10 U07.1 coding suggests that claims-based analyses may accurately capture patients with true COVID-19 disease. Epidemiological evaluations relying solely on U07.1 coding, however, may underestimate true disease burden. We acknowledge that SARS-CoV-2 RT-PCR is an imperfect "gold standard" and has itself had variable reported performance. ${ }^{6}$ We did not have access to corroborating information from other microbiological assessments or clinical presentations. Until higher fidelity testing is available, these data from a large 


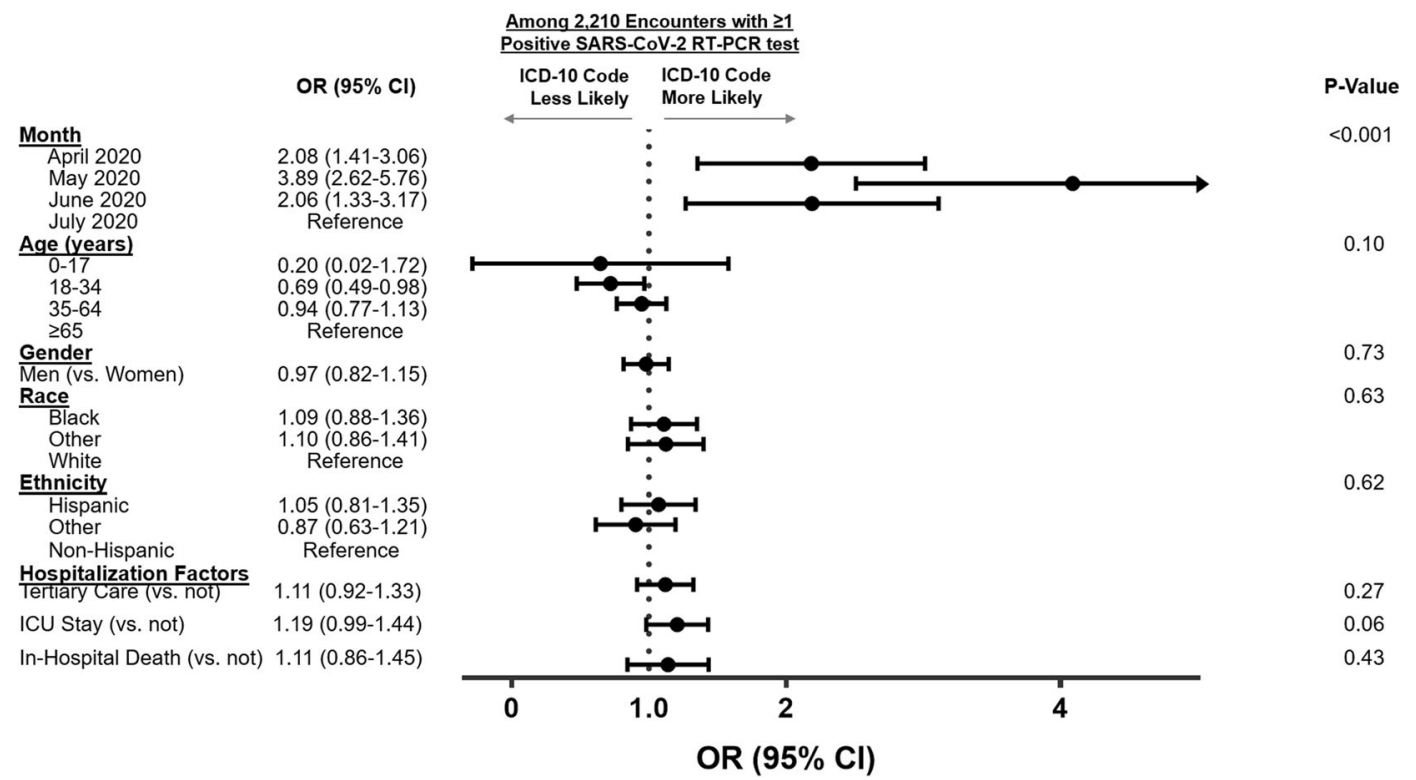

Figure 1 Independent predictors of ICD-10 sensitivity among encounters with $\geq 1$ positive SARS-CoV-2 RT-PCR test. CI, confidence interval; ICD-10, International Classification of Disease, $10^{\text {th }}$ revision; ICU, intensive care unit; OR, odds ratio; SARS-CoV-2 RT-PCR, severe acute respiratory syndrome coronavirus 2 reverse transcription polymerase chain reaction.

integrated health system inform the alignment between SARSCoV-2 RT-PCR testing and ICD-10 coding for COVID-19.

Access to Data. The first and corresponding authors, Drs. Ankeet S. Bhatt and Muthiah Vaduganathan, had full access to the study data and take responsibility for the integrity and accuracy of its analysis.

Ankeet S. Bhatt, $M D, M B A^{1}$

Erin E. McElrath, $M B A^{2}$

Brian L. Claggett, $P h D^{1}$

Deepak L. Bhatt, MD, MPH ${ }^{1}$

Dale S. Adler, $M D^{1}$

Scott D. Solomon, $M D^{1}$

Muthiah Vaduganathan, MD, $M P H^{1}$

${ }^{1}$ Division of Cardiology, Department of Medicine, Brigham and Women's Hospital, Heart \& Vascular Center and Harvard Medical School,

75 Francis St, Boston, MA 02115, USA

${ }^{2}$ Department of Medicine, Brigham and Women's Hospital,

Boston, MA, USA

Corresponding Author: Muthiah Vaduganathan, MD, MPH; Division of Cardiology, Department of Medicine, Brigham and Women's Hospital, Heart \& Vascular Center and Harvard Medical School, 75 Francis St, Boston, MA O2115, USA (e-mail: mvaduganathan@bwh.harvard.edu).

\section{Declarations:}

Conflict of Interest: Dr. Ankeet S. Bhatt has received consulting fees from Sanofi and is supported by the NHLBI T32 postdoctoral training grant (T32HLOO7604).
Dr. Claggett has received consultancy fees from Boehringer Ingelheim, Gilead, AOBiome, and Corvia.

Dr. Deepak L. Bhatt discloses the following relationships: Advisory Board: Cardax, Cereno Scientific, Elsevier Practice Update Cardiology, Level Ex, Medscape Cardiology, PhaseBio, PLx Pharma, Regado Biosciences; Board of Directors: Boston VA Research Institute, Society of Cardiovascular Patient Care, TobeSoft; Chair: American Heart Association Quality Oversight Committee; Data Monitoring Committees: Baim Institute for Clinical Research (formerly Harvard Clinical Research Institute, for the PORTICO trial, funded by St. Jude Medical, now Abbott), Cleveland Clinic (including for the ExCEED trial, funded by Edwards), Duke Clinical Research Institute, Mayo Clinic, Mount Sinai School of Medicine (for the ENVISAGE trial, funded by Daiichi Sankyo), Population Health Research Institute; Honoraria: American College of Cardiology (Senior Associate Editor, Clinical Trials and News, ACC.org: ViceChair, ACC Accreditation Committee), Baim Institute for Clinical Research (formerly Harvard Clinical Research Institute; RE-DUAL PCI clinical trial steering committee funded by Boehringer Ingelheim; AEGIS-II executive committee funded by CSL Behring), Belvoir Publications (Editor in Chief, Harvard Heart Letter), Duke Clinical Research Institute (clinical trial steering committees, including for the PRONOUNCE trial, funded by Ferring Pharmaceuticals), HMP Global (Editor in Chief, Journal of Invasive Cardiology), Journal of the American College of Cardiology (Guest Editor; Associate Editor), Medtelligence/ ReachMD (CME steering committees), Level Ex, MJH Life Sciences, Population Health Research Institute (for the COMPASS operations committee, publications committee, steering committee, and USA national co-leader, funded by Bayer), Slack Publications (Chief Medical Editor, Cardiology Today's Intervention), Society of Cardiovascular Patient Care (Secretary/Treasurer), WebMD (CME steering committees); Other: Clinical Cardiology (Deputy Editor), NCDR-ACTION Registry Steering Committee (Chair), VA CART Research and Publications Committee (Chair); Research Funding: Abbott, Afimmune, Amarin, Amgen, AstraZeneca, Bayer, Boehringer Ingelheim, Bristol-Myers Squibb, Cardax, Chiesi, CSL Behring, Eisai, Ethicon, Ferring Pharmaceuticals, Forest Laboratories, Fractyl, Idorsia, Ironwood, Ischemix, Lexicon, Lilly, Medtronic, Pfizer, PhaseBio, PLx Pharma, Regeneron, Roche, Sanofi Aventis, Synaptic, The Medicines Company; Royalties: Elsevier (Editor, Cardiovascular Intervention: A Companion to Braunwald's Heart Disease); Site Co-Investigator: Biotronik, Boston Scientific, CSI, St. Jude Medical (now Abbott), Svelte; Trustee: American College of Cardiology; Unfunded Research: FlowCo, Merck, Novo Nordisk, Takeda.

Dr. Solomon has received research grants from Alnylam. Amgen, AstraZeneca, Bellerophon, Bayer, BMS, Celladon, Cytokinetics, Eidos, Gilead, GSK, Ionis, Lone Star Heart, Mesoblast, MyoKardia, NIH/ NHLBI, Neurotronik, Novartis, Respicardia, Sanofi Pasteur, Theracos, 
and has consulted for Akros, Alnylam, Amgen, Arena, AstraZeneca, Bayer, BMS, Cardior, Cardurion, Corvia, Cytokinetics, Daiichi-Sankyo, Gilead, GSK, Ironwood, Merck, Myokardia, Novartis, Roche, Takeda, Theracos, Quantum Genetics, Cardurion, AoBiome, Janssen, Cardiac Dimensions, Tenaya, Sanofi-Pasteur, Dinaqor, Tremeau, CellProThera, Moderna.

Dr. Vaduganathan has received research grant support or served on advisory boards for American Regent, Amgen, AstraZeneca, Bayer AG, Baxter Healthcare, Boehringer Ingelheim, Cytokinetics, Lexicon Pharmaceuticals, and Relypsa, speaker engagements with Novartis and Roche Diagnostics, and participates on clinical endpoint committees for studies sponsored by Galmed and Novartis.

All other authors report no relevant disclosures to this work.

\section{REFERENCES}

1. Centers for Disease Control and Prevention. New ICD-10-CM code for the 2019 Novel Coronavirus (COVID-19), April 1, 2020. Available at: https:// www.cdc.gov/nchs/data/icd/Announcement-New-ICD-code-for-coronavirus-3-18-2020.pdf. Accessed August 15, 2020.
2. Centers for Disease Control and Prevention. ICD-10-CM Official Coding and Reporting Guidelines April 1, 2020 through September 30, 2020. Available at: https://www.cdc.gov/nchs/data/icd/COVID-19-guidelinesfinal.pdf. Accessed August 15, 2020.

3. Preliminary Medicare COVID-19 Data Snapshot | CMS. Available at: https://www.cms.gov/files/document/medicare-covid-19-data-snapshotmethodology.pdf. Accessed August 15, 2020.

4. McCarthy C, Murphy S, Cohen JA, et al. Misclassification of Myocardial Injury as Myocardial Infarction: Implications for Assessing Outcomes in Value-Based Programs. JAMA Cardiol. 2019;4:460-464.

5. Crabb BT, Lyons A, Bale M, et al. Comparison of international classification of diseases and related health problems, tenth revision codes with electronic medical records among patients with symptoms of coronavirus disease 2019. JAMA Netw Open 2020;3(8):e2017703.

6. Sethuraman N, Jeremiah SS, Ryo A. Interpreting Diagnostic Tests for SARS-CoV-2. JAMA 2020;323(22):2249-2251.

Publisher's Note: Springer Nature remains neutral with regard to jurisdictional claims in published maps and institutional affiliations. 